\title{
Article
}

\section{Shape-memory Nanofiber Meshes with Programmable Cell Orientation}

\author{
Eri Niiyamaa ${ }^{1,2}$, Kanta Tanabe ${ }^{1,3}$, Koichiro Uto ${ }^{4}$, Akihiko Kikuchi ${ }^{3}$ and Mitsuhiro Ebara ${ }^{1,2,3^{*}}$ \\ 1 International Center for Materials Nanoarchitectonics (WPI-MANA), National Institute for Materials \\ Science (NIMS), Tsukuba, Ibaraki 305-0044, Japan \\ 2 Graduate School of Pure and Applied Sciences, University of Tsukuba, Tsukuba, Ibaraki 305-8577, Japan \\ 3 Department of Materials Science and Technology, Tokyo University of Science, 6-3-1 Niijuku, Katsushika, \\ Tokyo 125-8585, Japan \\ 4 International Center for Young Scientists (ICYS), National Institute for Materials Science (NIMS), Tsukuba, \\ Ibaraki 305-0044, Japan \\ * Correspondence: EBARA.Mitsuhiro@nims.go.jp
}

\begin{abstract}
This paper reports a rational design of temperature-responsive nanofiber meshes with shape-memory effect. The meshes were fabricated by electrospinning a poly( $\varepsilon$-caprolactone) (PCL)based polyurethane with different contents of soft and hard segments. The effects of PCL diol/hexamethylene diisocyanate (HDI)/1,4-butanediol (BD) molar ratio in terms of the contents of soft and hard segments on the shape-memory properties were investigated. Although the mechanical property improved with increasing hard segment ratio, optimal shape-memory properties were obtained with a PCL/HDI/BD molar ratio of 1:4:3. At a microscopic level, the original nanofibrous structure was easily deformed into a temporary shape, and recovered its original structure when the sample was reheated. A higher recovery rate $(>89 \%)$ was achieved even when the mesh was deformed up to $400 \%$. Finally, the nanofiber meshes were used to control the alignment of human mesenchymal stem cells (hMSCs). The hMSCs aligned well along the fiber orientation. The proposed nanofibrous meshes with the shape-memory effect have the potential to serve as in vitro platforms for the investigation of cell functions as well as implantable scaffolds for wound-healing applications.
\end{abstract}

Keywords: shape memory nanofiber; shape memory polymer; poly(e-caprolactone); melting temperature; cell orientation; polyurethane

\section{Introduction}

Stimuli-responsive fibrous materials with shape-memory effect (also called "shape-memory fibers") are receiving great attention for potential application in the fields of biomedical, filtration, robotics, catalysis, etc. [1-7]. Shape-memory polymers (SMPs) are a class of temperature-responsive polymers that can change from a temporary shape to a memorized permanent shape upon application of heat. The glass transition temperature $\left(T_{\mathrm{g}}\right)$ or melting temperature $\left(T_{\mathrm{m}}\right)$ is commonly used as the shape-switching temperature. The use of $T_{\mathrm{m}}$ is more favorable for creating better SMPs because the enthalpy changes of the solid-liquid phase transition are much larger than that of the glass-rubber transition or liquid crystalline transition. Poly( $\varepsilon$-caprolactone) (PCL)-based SMPs were widely studied in the past decades especially in the field of biomaterials because of their potential biocompatibility [8] and biodegradability [9-10]. In addition, the development of various fabrication processes for SMPs has resulted in diverse structures. For example, SMPs can be classified as shapememory films, foams, particles, surfaces, and fibers [4, 11-17]. Particularly, SMPs in the form of fibers are generating great interests in structural and functional applications owing to their extremely high surface area, porous structure, and filtration/penetration properties [18-21]. Various thermoplastic polymers have been spun into nanofibers by the electrospinning method, which has been extensively acknowledged as an efficient and convenient approach for producing nanofibrous materials [22,23]. Fibrous materials are attractive as tissue engineering scaffolds because of their inherent advantages 
such as cell attachment, controlled porous architecture, and a 3-D microenvironment for cell-cell contact. Fibrous scaffolds have been applied to cardiovascular tissue engineering [24,26], musculoskeletal tissue engineering [27,28], neural tissue engineering [29], and stem cell engineering [30,31]. In addition, submicron-diameter fibers can provide tissue-matching mechanical compliance and stronger topographic cues by mimicking the extracellular matrix (ECM). Recent studies have revealed that the topography of ECMs strongly affects the cellular response to differentiation and production of tissues [32-35].

Because of the above advantages, nanofibrous materials with shape-memory effect have been increasingly investigated. Matsumoto et al. prepared shape-memory microfibers using poly $(\omega-$ pentadecalactone) and PCL, and achieved a strain recovery rate of $>89 \%$ and a strain fixity rate of $>82 \%$ by applying small deformations ( $25 \%)$ [36]. Fejös et al. fabricated triple-shape memory nanofibers using the $T_{\mathrm{g}}$ of epoxy and the $T_{\mathrm{m}}$ of PCL as the shape-switching temperatures [37]. Good strain recovery rates $\left(>94 \%\right.$ for $T_{\mathrm{g}}$ and $>89 \%$ for $T_{\mathrm{m}}$ ) were obtained although the samples were deformed to only $2 \%$ strain. Ji et al. developed a series of shape-memory polyurethanes with varying hard-soft segment ratios [38]. The shape fixity ratios increased from $75 \%$ to $92 \%$ and the shape recovery ratios decreased from $92 \%$ to $85 \%$ with increasing hard segment contents. Barmouz et al. investigated the shape memory behavior of poly(lactic acid)-thermoplastic polyurethane/cellulosenanofiber bio-nanocomposites. They resulted that adding cellulose nanofibers to the polymeric matrix imposed notable promotion of stress recovery (over $40 \%$ ) with a slight reduction in strain recovery [39]. Kawaguchi et al. investigated the effects of the addition of chitosan fiber (biomass nanofiber made by Sugino (BiNFi-s)) to polyether-based thermoplastic polyurethane (TPU) on material properties. The addition of BiNFi-s or glass fiber to TPU did not influence the glass transition temperature, although the crystal structure changed from semi-crystalline to amorphous. The elastic modulus increased $40 \%$ by the addition of $5 \mathrm{wt} \%$ BiNFi-s ( $2.31 \mathrm{MPa}$ ) compared with plain TPU (1.65 $\mathrm{MPa})$, and these composites exhibited shape recovery with clinically relevant changes in temperature [40]. Although these values are considered good for the shape-memory behavior, shape-memory nanofibers generally show a relatively lower shape fixity and recovery properties compared to a bulk shape-memory film. One of the major reasons is its polymer network architecture. In general, SMP systems can be broadly classified into two types based on the network architecture: (1) a physically cross-linked network and (2) a covalently cross-linked network. Because it is difficult to cross-link the networks chemically during electrospinning, physical cross-linking is more suitable for electrospun nanofiber systems. However, compared with chemical cross-linking, physical crosslinking generally results in less structurally stable networks.

In this study, we report the rational design of shape-memory nanofiber meshes with higher shape-memory ability using PCL-based polyurethane by electrospinning. A series of polyurethanes with different soft and hard segment contents was prepared. Hard segments can bind themselves via hydrogen bonding and crystallization, making the polymer very solid. On the other hand, soft segments show reversible phase transformation at the $T_{\mathrm{m}}$, which is responsible for the shape-memory effect. The effects of hard and soft segment contents on the fiber processability, mechanical properties, $T_{\mathrm{m}}$, and fiber stability were evaluated. Systematic variations of these parameters will help identify key compositional features essential for a high shape-memory ability. Particularly, samples were subjected to higher deformations ( 400\%) in this study. In addition, we examined the control of cell orientation on the nanofiber meshes with different fiber alignments. The polymer design strategy applied in this study can increase the prospective applications of shape-memory fibers in the biomedical field.

\section{Results}

\subsection{Fabrication of PCL-based polyurethane nanofiber meshes}

First, PCL diol was synthesized by the ring-opening polymerization of $\mathrm{CL}$ in the presence of a catalyst and an initiator $[2,12,14]$. Then, PCL-based polyurethane was synthesized from PCL 
diol using different ratios of 1,4-butanediol (BD) and hexamethylene diisocyanate (HDI) (Scheme 1). In this study, three types of PCL-based polyurethanes were synthesized with soft:hard segment ratios of 1:5, 1:7, and 1:9 (PCL:HDI:BD = 1:3:2, 1:4:3, and 1:5:4, respectively). The successful synthesis of PCL-based polyurethane was confirmed by ${ }^{1} \mathrm{H}$ NMR spectroscopy and gel permeation chromatography (GPC) (Figure S1 and Table 1). Approximately, 6.8, 9.2, and 11.3\% of hard segments were introduced into the PCL-based polyurethanes with soft:hard segment ratios of 1:5, 1:7, and 1:9, respectively. The samples were abbreviated as PCL-6.8, PCL-9.2, and PCL-11.3, respectively. The number-average molecular weight $\left(M_{n}\right)$ of the PCL-based polyurethane was found to be approximately 35,000. Molecular weight is an important factor for electrospinning. A lower molecular weight results in the formation of beads or particles because of insufficient molecular chain entanglement in the fiber fabrication process, wherein the polymer solution is jetted to a collector. Figure S2 shows the morphology of the electrospun PCL with a molecular weight of 5,000. Our previous report also suggested that polymers with $M_{n}<10,000$ formed bead-like structures, while polymers with a relatively higher $M_{n}(>50,000)$ formed fibers via electrospinning at a lower solution concentration [41].
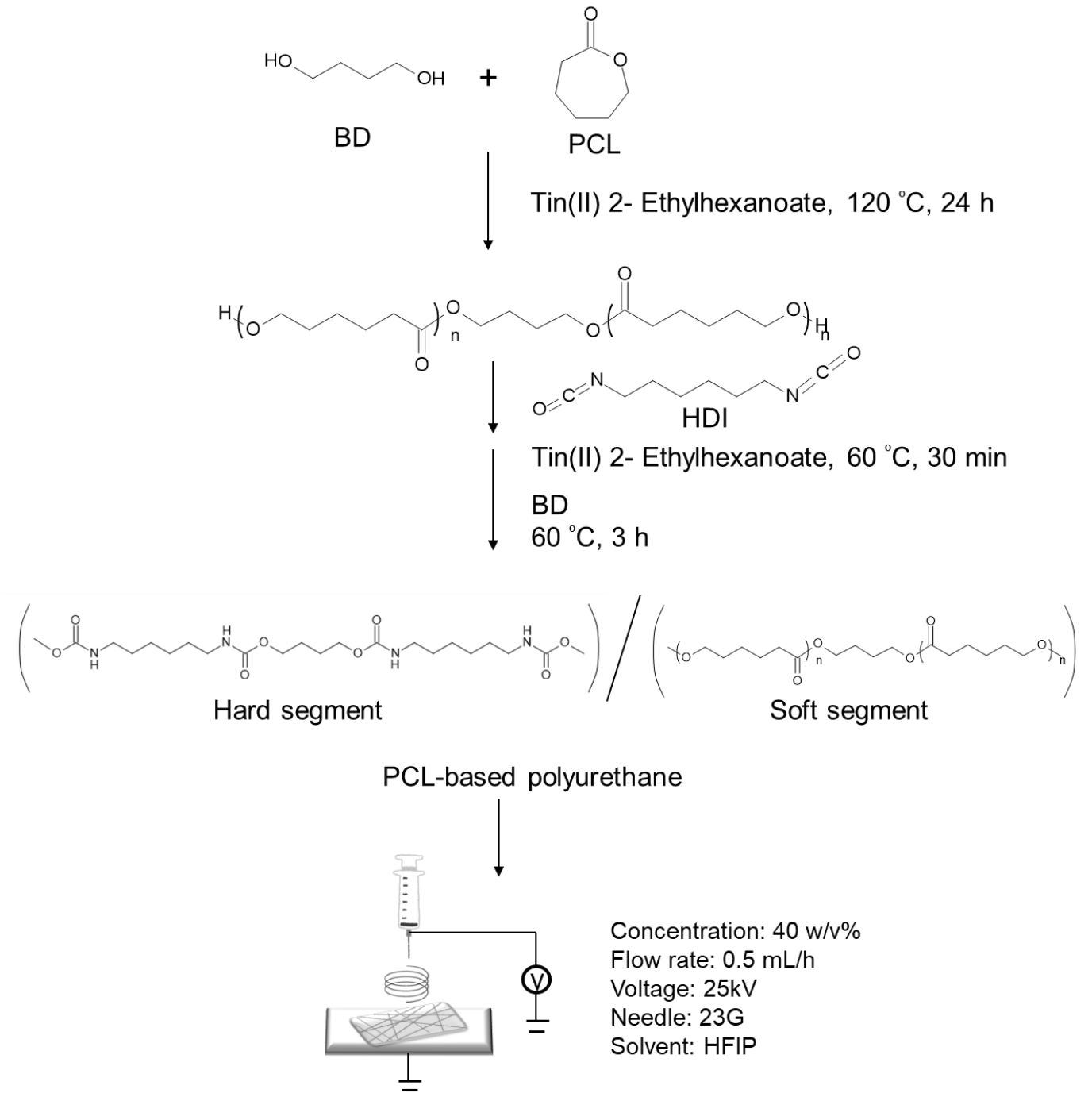

Electrospinning

Scheme 1. Synthesis of PCL-based polyurethanes and fabrication of nanofiber mesh by electrospinning. 
Table 1. Characteristic data of a series of PCL-based polyurethanes

\begin{tabular}{|c|c|c|c|c|c|c|c|c|}
\hline \multirow[t]{2}{*}{ Samples } & \multirow{2}{*}{$\begin{array}{l}\text { Composition } \\
\text { (Molar ratio) } \\
\text { PCL : HDI : BD }\end{array}$} & \multicolumn{2}{|c|}{$\begin{array}{c}\text { Segment ratio1) } \\
\text { (Molar ratio) }\end{array}$} & \multicolumn{2}{|c|}{$\begin{array}{l}\text { Segment ratio } \\
\qquad(w / w \%)\end{array}$} & \multicolumn{2}{|c|}{$\begin{array}{l}\text { Molecular } \\
\text { weight }^{2)}\end{array}$} & \multirow{2}{*}{$\begin{array}{c}\left.\mathrm{PDI}^{2}\right) \\
\left(M_{\mathrm{w}} / M_{\mathrm{n}}\right)\end{array}$} \\
\hline & & Soft & Hard & Soft & Hard & $M_{w}$ & $M_{n}$ & \\
\hline PCL & - & - & - & - & - & 59,700 & 46,300 & 1.29 \\
\hline PCL-6.8 & 1:3:2 & 1 & 5 & 93.2 & 6.8 & 78,900 & 54,000 & 1.46 \\
\hline PCL-9.2 & $1: 4: 3$ & 1 & 7 & 90.8 & 9.2 & 73,200 & 53,000 & 1.38 \\
\hline PCL-11.3 & $1: 5: 4$ & 1 & 9 & 88.7 & 11.3 & 93,500 & 68,000 & 1.37 \\
\hline
\end{tabular}

1)Soft:Hard = PCL:HDI + BD

2)Measured by GPC.

In addition to molecular weight, solution concentration, applied electric field, and distance between the spinneret and collectors are the factors affecting the electrospinning process. Therefore, the nanofiber meshes were fabricated under optimized conditions, as described in the materials section. All samples showed good processability at a concentration of $40 \mathrm{w} / \mathrm{v} \%$. As shown in Figure 1 , smooth fibrous structures without beads were formed with average diameters of $417 \pm 20,511 \pm 30$, and $1329 \pm 61 \mathrm{~nm}$ for PCL-6.8, PCL-9.2, and PCL-11.3, respectively. Interestingly, polymers with extremely low $(<4.4 \%)$ or high $(>21.3 \%)$ hard segment contents were difficult to form into fibers. Particularly, electrospinning of PCL-21.3 resulted in a bead-like structure rather than a fiber mesh (Figure S3). Besides polymer composition, polymer architecture is also considered an important factor in electrospinning. Figure S4 shows the SEM images of the electrospun fibers from the fourbranched PCL. Even though the molecular weight is similar, no fiber structures were formed.
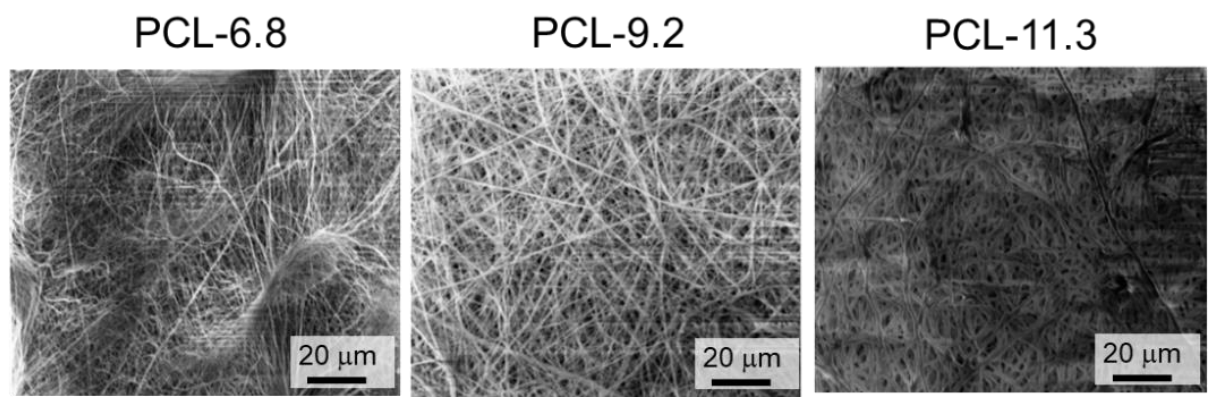

Figure 1. SEM micrographs of fibers electrospun from PCL-6.8, 9.2, and 11.3 (10 kV accelerating voltage).

\subsection{Thermal properties}

The thermal properties of the nanofibers were characterized by DSC. Figure 2 shows the DSC curves of the nanofibers with different hard-to-soft segment ratios. As can be seen, the $T_{\mathrm{m}}$ of the nanofiber meshes is slightly lower than that of the corresponding pure PCL. In general, the $T \mathrm{~m}$ of cross-linked PCL is lower than that of an uncrosslinked one because the addition of cross-linking netpoints disrupt the crystallization of polymer segments [42]. Therefore, the DSC result indicates that hydrogen bonds were successfully formed between the hard segments. Particularly, in the case of nanofibers, this trend becomes more significant because the molecular orientation generated during the spinning process can lead to the alignment of polyurethane molecules along the fiber axis. The preferred orientation of the molecules leads to the packing and aggregation of hard segments into hard-segment microdomains. As a result, the $T_{\mathrm{m}}$ becomes lower than that of the linear PCL. 


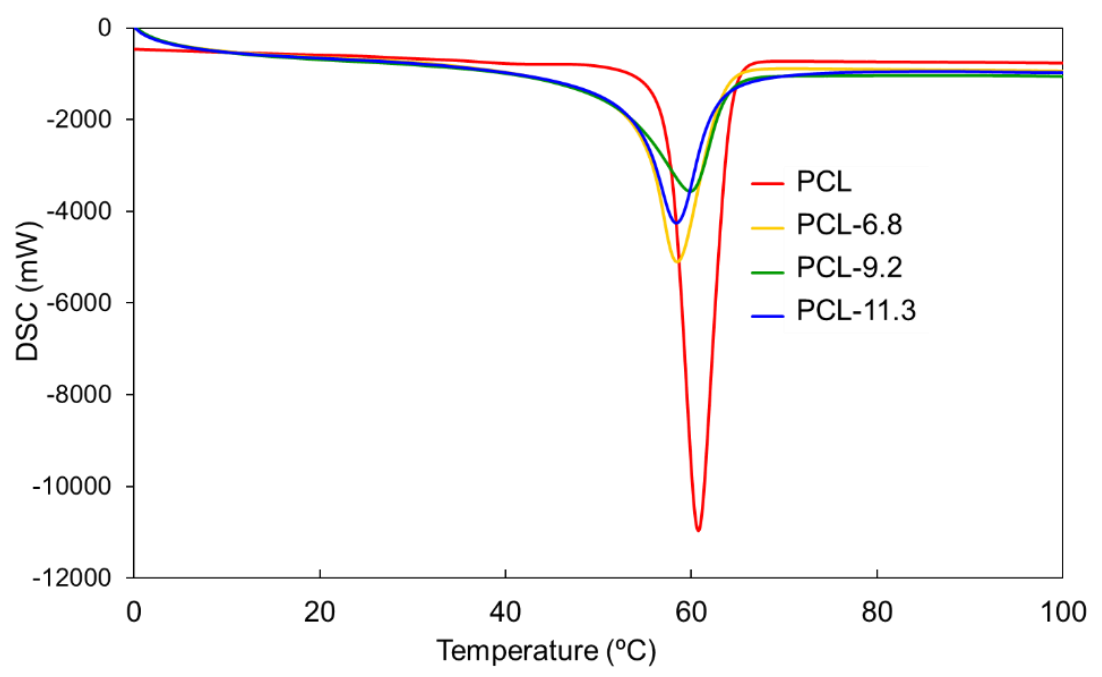

Figure 2. DSC curves of electrospun fibers of pure PCL and PCL-6.8, 9.2, and 11.3.

To confirm the thermostability of the physically cross-linked polyurethane samples, the samples were heated to near their $T_{\mathrm{m}}$ (around $60^{\circ} \mathrm{C}$ ) and their morphologies were analyzed by SEM. Figure 3 presents the SEM images before and after heating. Before heating, all the nanofibers showed the formation of uniform fibers. After heating, PCL-6.8 melted, whereas PCL-9.2 and PCL-11.3 retained their fibrous morphologies. This result indicates that the physical cross-linking within PCL-6.8 was insufficient to maintain its morphology above the $T_{\mathrm{m}}$. On the other hand, PCL-21.3 $(>21.3 \%)$ broke after heating even though it had the highest hard segment content (Figure S5). These results suggest that careful selection of a hard-to-soft segment ratio is significant for designing high-performance shape-memory nanofibers.

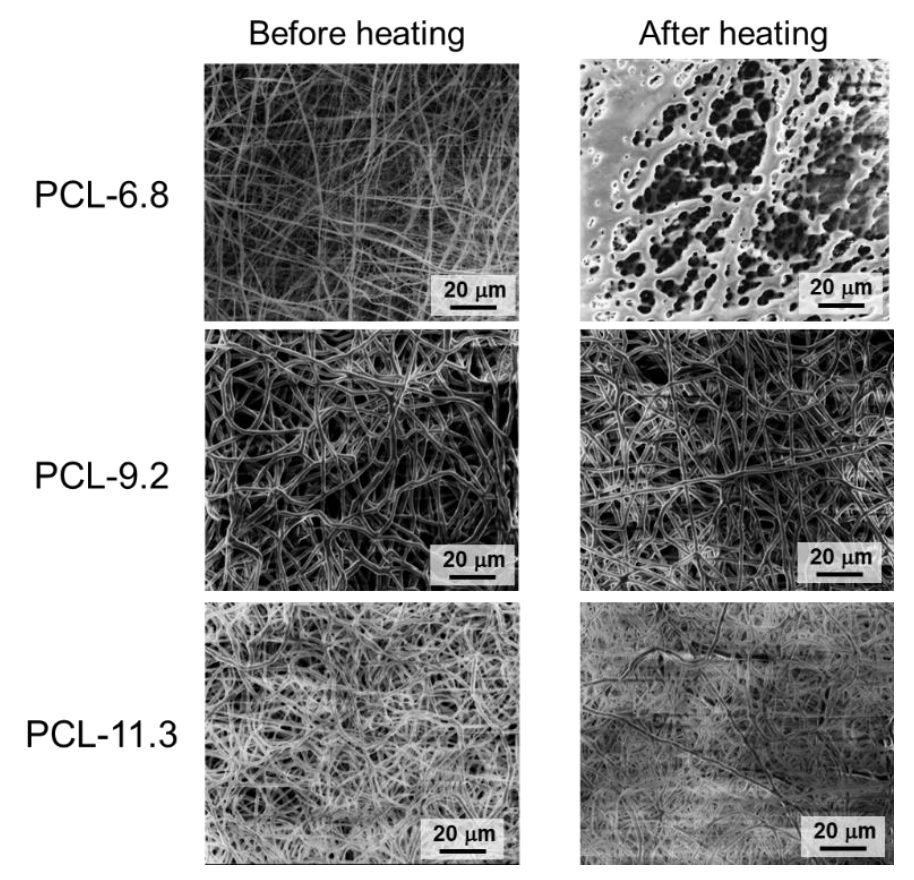

Figure 3. SEM micrographs of electrospun fibers of PCL-6.8, 9.2, and 11.3 before and after heating at $60^{\circ} \mathrm{C}$ for $24 \mathrm{~h}$. 


\subsection{Shape-memory properties}

The shape memory capability of the nanofiber meshes was assessed by SEM and orientation analyses. Figure 4(a, top) shows the digital camera images of PCL-9.2 nanofibers before deformation (left image), after 300\% deformation and fixation (middle image), and after shape recovery (right image). The corresponding microscopic shape changes are shown in Figure 4(a, middle). The fibers showed different structures before and after deformation. The original, randomly oriented fibrous structure (left image) was easily deformed into a temporary shape (middle image), and the fibers tended to orient along the strain direction. The orientated fibers recovered their original structure when the sample was reheated. Figure 4(a, bottom) shows the cross-sectional images of PCL-9.2 nanofibers during the shape memory cycle. The fiber diameters slightly decreased from around 600 $\mathrm{nm}$ to $500 \mathrm{~nm}$ after stretching (Figure $4 \mathrm{~b}$ ), and the cross-sectional shape changed slightly from circular to ellipsoidal after stretching. After shape recovery, the fiber diameters recovered to around $700 \mathrm{~nm}$. Nevertheless, the nanofiber structure was stable after the shape memory test cycles at a microscopic level. Figure 4(c) presents a comparison between the strain fixity rate $\left(R_{\mathrm{f}}\right)$ and strain recovery rate $\left(R_{\mathrm{r}}\right)$ of the PCL-9.2 nanofiber mesh under different deformation rates $(200,300$, and $400 \%)$. The $R_{f}$ values were 88,90 , and $96 \%$, and the $R_{r}$ values were 100,89 , and $91 \%$ after 200,300 , and $400 \%$ deformation, respectively.

(a)

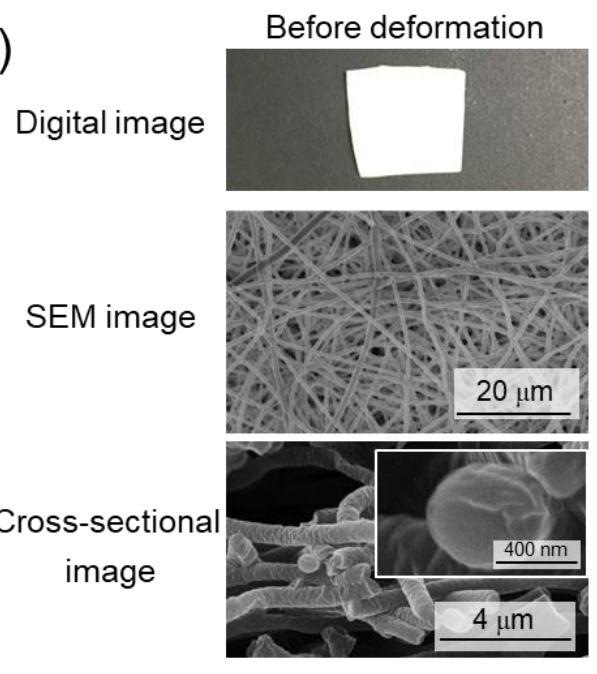

(b)

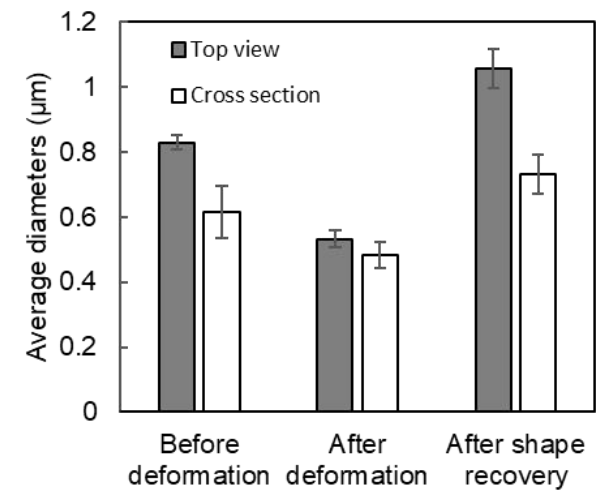

After deformation
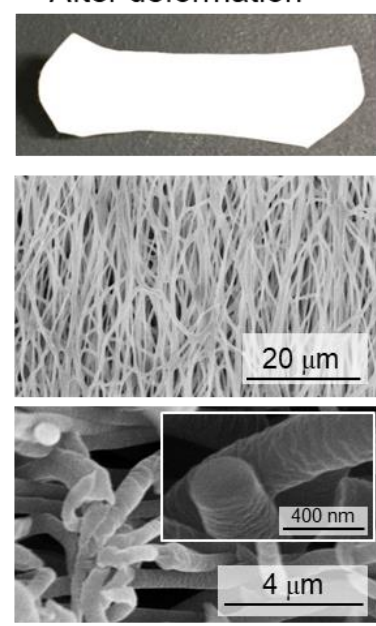

(c)

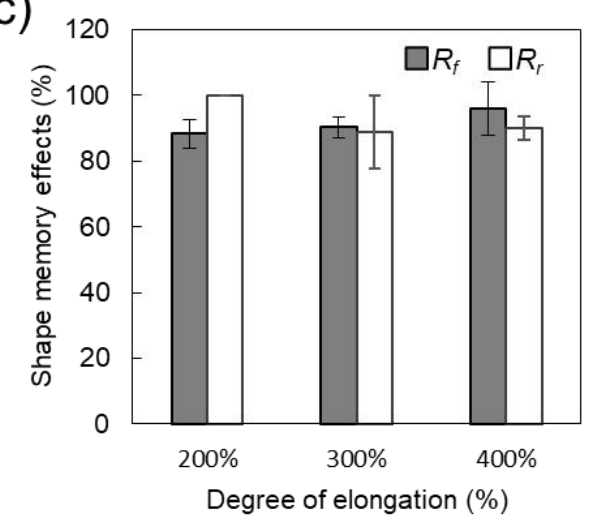

Figure 4. Evaluations of shape-memory effect of PCL-9.2 nanofibers. (a) Digital (top) and SEM (middle and bottom) images of PCL-9.2 nanofibers before deformation (left), after deformation (middle), and after shape memory recovery (right). (b) Average diameters of PCL-9.2 nanofibers calculated from the cross-sectional images before deformation, after deformation, and after shape memory recovery $\left(\mathrm{n}=3,{ }^{*} \mathrm{p}<0.001,{ }^{* *} \mathrm{p}<0.002\right)$. (c) Shape fixity rate $\left(R_{\mathrm{f}}\right)$ and recovery rate $\left(R_{\mathrm{r}}\right)$ of PCL9.2 nanofibers under different elongation degrees $(200,300$, and $400 \%)(n=3)$. 
To visualize the fiber orientation during the shape memory cycle, orientation analysis was performed using image analysis software. The software evaluated the structure tensor of each Gaussian-shaped window by computing the continuous spatial derivatives in the $x$ and $y$ dimensions using Gaussian interpolation. For qualitative visual representation of the orientation, the grey-scale SEM images were converted into color-coded images (Figure 5(a)). The fibers oriented along the deformation direction were defined to have a $\pm 90^{\circ}$ orientation. The fiber orientation was clearly observed. For quantitative assessment, the average population of different orientation distributions of the fibers is presented in Figure 5(b).

(a)
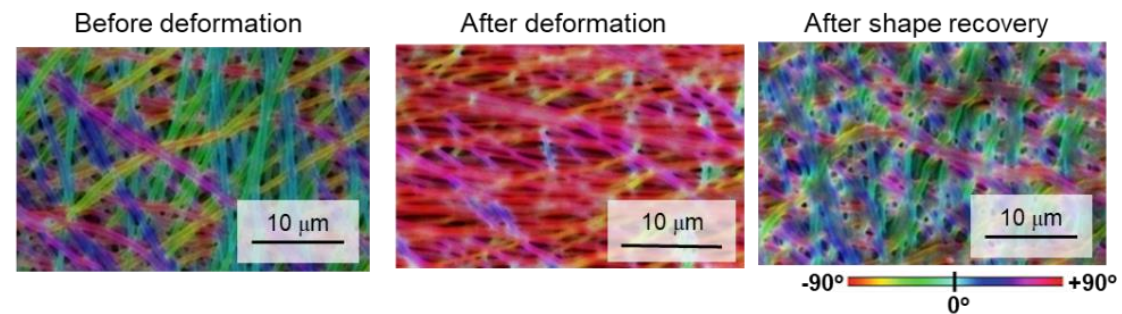

(b)

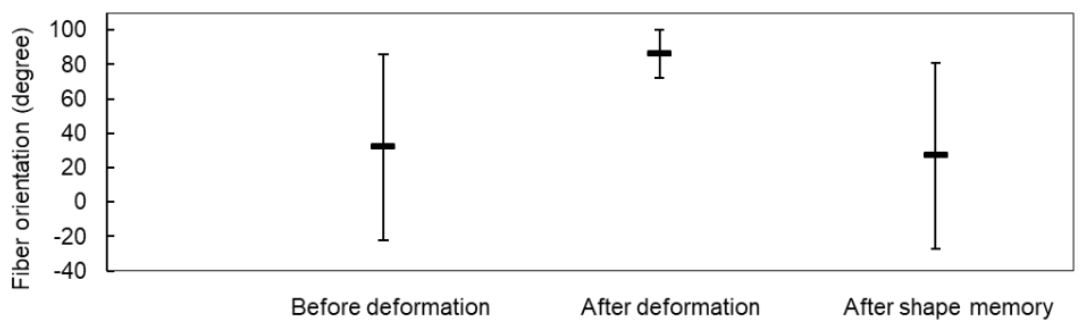

Figure 5. Orientation analysis of PCL-9.2 nanofibers before deformation, after deformation, and after shape memory recovery. (a) SEM images analyzed by Image J software for orientation evaluation and (b) fiber orientation distribution of nanofibers before deformation, after deformation, and after shape memory.

\subsection{Cellular alignment}

Cells are sensitive to topographical cues via changes in cell morphology. Recent studies have demonstrated that stem cells such as mesenchymal stem cells (MSCs) can detect the biomechanical properties of the underlying substrate and alter their morphology accordingly via rearrangement of the cytoskeletal components such as actin structures and focal adhesion complexes, thus facilitating the conversion of extrinsic mechanical signals into the initiation of intracellular signaling [17, 43]. Therefore, we assessed the potential of our shape-memory nanofiber meshes to influence the MSC morphology. Human mesenchymal stem cells (hMSCs) were seeded onto the PCL-9.2 nanofiber mesh with and without deformation $(300 \%)$. The cells on the random fiber mesh (non-stretched) spread extensively but with a random orientation (Figure 6), whereas the cells on the aligned fiber mesh (stretched) elongated along the fiber axis. Since the average diameter of an individual fiber is substantially narrower than the dimensions of a cell, hMSCs cannot spread in an unrestricted manner on the fiber meshes as they would on a planar surface. As a result, the intracellular cytoskeletal structure of hMSCs possibly altered as compared with those on the planar surfaces, most notably resulting in cellular alignment along the dominant fiber axis. In this study, we cultured cells on the sample before or after shape-memory activation. Therefore, the effect of temperature was not considered. However, to observe how cells respond to dynamic change of substrate during shapememory activation is more attractive from the viewpoint of mechanobiology study. Therefore, the next step of this study is to adjust the shape-switching temperature of fibers to $37^{\circ} \mathrm{C}$ and examine dynamic cell culture on them. 


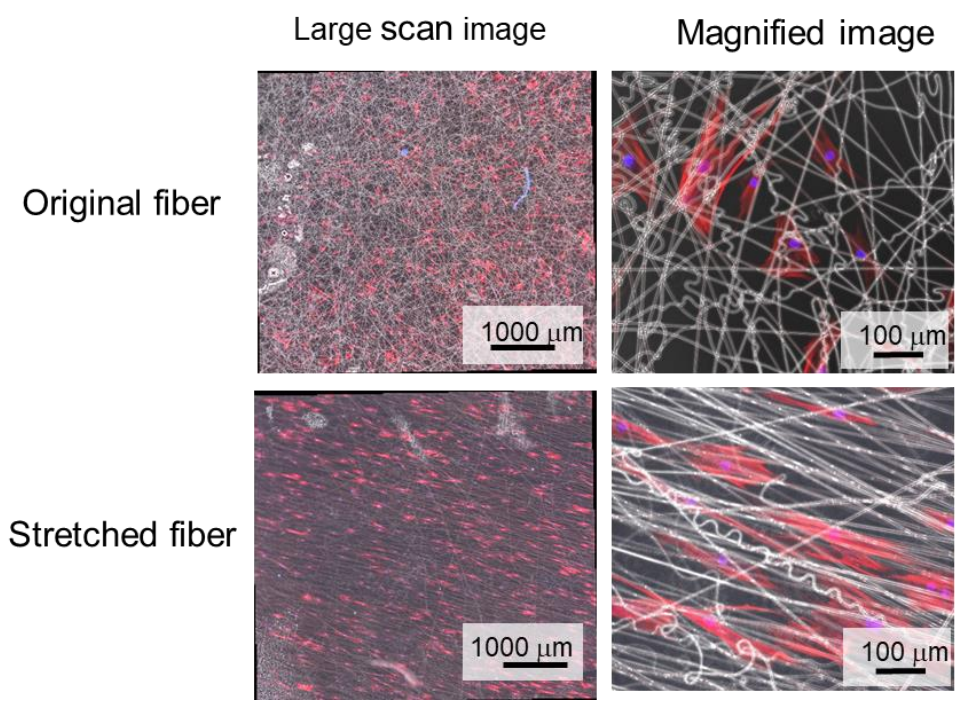

Figure 6. Adhesion morphology and alignment of human mesenchymal stem cells (hMSCs) on original (left) and stretched (right) PCL-9.2 nanofibers. hMSCs on each fiber type were stained with f-actin (red) and nucleus (blue). hMSCs were cultured at $37^{\circ} \mathrm{C}$ for $24 \mathrm{~h}$. Large -area scan (left) and magnified (right) images show global and local alignment of hMSCs.

\section{Discussion}

SMPs have the ability to recover their permanent shape from a temporary shape under an external stimulus. Among the SMPs, thermally induced SMPs have been extensively investigated. We recently developed a thermally induced SMP using a cross-linked PCL, which has an adjustable shape-switching temperature near the biologically relevant temperatures [12-14, 16]. Such SMPs enable the development of shape-memory cell culture substrates [12-14, 16]. The shape-memory nanopatterns are programmed by mechanically embossing the desired topography into the polymer surface. Previous studies have demonstrated that cells can reorganize their cytoskeletons after shape transition. In addition, the study demonstrated that the contractile direction of cardiomyocyte sheets changed by shape-memory transitions [35]. These studies have drawn much attention as they provide a new category of dynamic cell culture platforms for fundamental research and practical applications. Such field is called "mechanobiology". In mechanobiology field, the cell-extracellular matrix interactions are considered to be important extrinsic factors that regulate cell fate. In other words, cellular mechanisms are described whereby cells could sense, measure and respond to the space they were in, in particular to the rigidity of the surface the cells were on. Therefore, the manipulation of topographical cues has a strong potential as a strategy for stem cell engineering and regenerative medicine by mimicking changing physiological conditions during would healing, organogenesis, and cancer metastasis. We also developed shape-memory microparticles by an in situ oil-in-water emulsion polymerization technique [15]. The particles with a disk-like temporal shape recovered their original spherical shape upon heating. Such particles can be potentially used for bioseparation, and in future, for drug delivery and immune engineering. In this study, shape-memory nanofiber meshes were prepared by electrospinning. Interestingly, polymers with extremely low or high hard segment contents were difficult to form into fibers because the inter-/intra-polymer interactions or entanglements were too low or too high, respectively. Smooth fibrous structures were successfully formed from PCL-6.8, 9.2, and 11.3, although PCL-6.8 melted after being heated above the $T_{m}$ because the physical cross-linking within PCL-6.8 was insufficient to maintain its morphology.

To discuss the detailed mechanism of the shape-memory nanofibers, the microscopic shapememory properties were considered. The hard segments as the fixable phase determined the 
permanent shape of the polymer network, and the soft segments as the reversible phase decided the molecular switching. If the working temperature is higher than the $T_{m}$, the crystalline region of the molecular chains will melt. If the nanofiber meshes are stretched, the molecular chains will extend. Moreover, if the sample is cooled to below its $T_{m}$ and the fiber is kept at a constant strain, the molecular chains will crystallize. As a result, the internal stress is stored in the fibers and the associated deformation is fixed temporally. After reheating to above the $T_{m}$, the molecular chains become flexible and the nanofiber mesh recovers its original shape. Therefore, shape fixity increased, and shape recovery ratios decreased gradually with increasing hard segment content. In our experiments, the microscopic change in the fiber structure was successfully observed by SEM after the fibers were subjected to deformation and recovery processes. The microscopic morphological changes based on the shape-memory effect in all the fibers are almost the same as the macroscopic changes. These results indicated that the shape-memory properties of the nanofibers were substantially determined by the internal structure of the PCL-based polyurethane but not by the macroscopic structural changes within the non-woven fabric. Compared to other shape-memory forms, many factors need to be considered to design shape-memory nanofibers. For example, fibrous materials have higher porosity, which can provide a low shape fixity ratio. It has also been proven that the electrospinning process produces a partial molecular orientation that can lead to an increase in the amount of the hard-segment phase, resulting in an increased recovery stress. However, the increase in the recovery stress adversely affected the fixing of the temporary shape, and thus, the shape fixity decreased. We also evaluated the recovery stress of PCL-9.2 after shape-memory activation. The value obtained from a tensile test was around $11 \mathrm{MPa}$. This value is relevant to previously reported value $[38,44]$.

Thus, such unique and tunable aspects of shape-memory nanofibers make them useful for many biomedical applications. For example, their rapid recovery could enable their application as stents and endovascular thrombectomy devices in surgical approaches. In contrast, a slower recovery rate would provide an opportunity to investigate the long-term influence of dynamic changes in matrix structures on cell behaviors such as cell differentiation and lineage specification. On a related point, it should be noted that the present study only demonstrates cell culture on the shape-memory meshes before and after deformation. However, we anticipate that the cell behavior during the dynamic shape-memory changes can be investigated in a future study.

\section{Materials and Methods}

\subsection{Materials}

HDI, BD, CL, and 1,1,1,3,3,3-hexafluoro-2-propanol (HFIP) were obtained from Tokyo Chemical Industry Co., Ltd. (Tokyo, Japan). Tin (II) 2-ethylhexanoate, rhodamine phalloidin, 4',6-diamidine-2phenylindole dihydrochloride (DAPI), fibronectin, and $0.1 \%$ triton X-100 were purchased from Sigma-Aldrich Japan (Tokyo, Japan). Phosphate buffered saline (PBS) was purchased from Nakalai Tesque (Kyoto, Japan). Xylene, n-hexane, diethylether, tetrahydrofuran (THF), and paraformaldehyde were obtained from FUJIFILM Wako Pure Chemical Corporation (Osaka, Japan). Human mesenchymal stem cells (hMSCs) were purchased from Lonza (Basel, Switzerland).

\subsection{Polymerization and characterization}

$\mathrm{BD}(194 \mu \mathrm{L}, 2.2 \mathrm{mmol})$ as an initiator was dried in vacuum overnight in a $300 \mathrm{~mL}$ round-bottom flask. CL ( $46.3 \mathrm{~mL}, 0.44 \mathrm{~mol})$ was added into the flask and stirred under a $\mathrm{N}_{2}$ atmosphere. Five drops of tin (II) 2-ethylhexanoate as a catalyst ( 5 droplets, $0.5 \mathrm{mmol}$ ) was then dropwise added and stirred at $120^{\circ} \mathrm{C}$ for $24 \mathrm{~h}$. The product was then completely dissolved in THF. The obtained PCL was purified by reprecipitation from hexane and diethyl ether. Then, $800 \mathrm{mg}$ of purified PCL and $20 \mathrm{~mL}$ of xylene 
were added into a $50 \mathrm{mg}$ sample tube and stirred at $60^{\circ} \mathrm{C}$ for $15 \mathrm{~min}$. HDI and tin (II) 2-ethylhexanoate were then added into the mixture and stirred at $60^{\circ} \mathrm{C}$ for $30 \mathrm{~min}$ followed by the addition of BD. The added amounts of HDI and BD were $44 \mu \mathrm{L}(0.27 \mathrm{mmol})$ and $16 \mu \mathrm{L}(0.18 \mathrm{mmol}), 58 \mu \mathrm{L}(0.36 \mathrm{mmol})$ and $24 \mu \mathrm{L}(0.27 \mathrm{mmol})$, and $72.5 \mu \mathrm{L}(0.45 \mathrm{mmol})$ and $32 \mu \mathrm{L}(0.36 \mathrm{mmol})$ to obtain PCL:HDI:BD molar ratios of 1:3:2, 1:4:3, and 1:5:4 (soft:hard segment $=1: 5,1: 7$, and 1:9), respectively. After stirring at $60^{\circ} \mathrm{C}$ for $3 \mathrm{~h}$, the mixture was purified by reprecipitation with a mixed solution of hexane and chloroform (80:3). The obtained polymer was filtered and dried in vacuum. The structures were determined by ${ }^{1} \mathrm{H}$-NMR spectroscopy (JEOL, Tokyo, Japan) with $\mathrm{CDCl}_{3}$ as a solvent (Figure S1). The molecular weights were determined by GPC equipped with TSKgel G4000Hhr and TSKgel G3000Hhr columns and a refractive detector using $\mathrm{N}, \mathrm{N}$-dimethylformamide (DMF) with $10 \mathrm{mM} \mathrm{LiCl}$ as the eluent and solvent $\left(0.8 \mathrm{~mL} / \mathrm{min}, 40^{\circ} \mathrm{C}\right)(\mathrm{HLC}-8220 \mathrm{GPC}$, Tosho Corporation, Tokyo, Japan) (Table 1$)$.

\subsection{Electrospinning method and characterization of nanofibers}

The electrospinning solution was prepared by dissolving the polymer in HFIP (40 w/v\%). PCL with different ratios of HDI and BD were electrospun into nanofibers using an applied voltage of 25 $\mathrm{kV}$, a needle gauge of 23, a flow rate of $0.5 \mathrm{~mL} / \mathrm{h}$, and a $15 \mathrm{~cm}$ separation between the needle and the collector plate (Nanon-01A, MECC Co., Ltd., Japan). The formation of electrospun nanofibers was observed using a scanning electron microscope (SEM; SU8000, Hitachi High-Technologies Corporation, Tokyo, Japan). The thermal property of the nanofibers with different ratios of HDI and BD was measured by differential scanning calorimetry (DSC; 6100, SEIKO Instruments, Chiba, Japan) at a heating rate of $5{ }^{\circ} \mathrm{C} / \mathrm{min}$. For the thermal stability test, a nanofiber mesh with a soft:hard segment ratio of 1:5, 1:7, or $1: 9$ was placed in an oven at $60^{\circ} \mathrm{C}$ for $24 \mathrm{~h}$. The nanofiber morphology before and after heating was compared by SEM observation. Because polyurethanes are known to hygroscopic material, we have conducted all experiments under constant temperature/humidity conditions $\left(21^{\circ} \mathrm{C} / 25 \%\right)$.

\subsection{Shape memory behavior}

The shape memory effect of the electrospun nanofibers with a soft:hard segment ratio of 1:7 was evaluated in terms of morphology, diameter change, orientation, and shape recovery rate of the nanofibers before deformation, after deformation, and after shape memory recovery. The nanofiber was first heated at $60^{\circ} \mathrm{C}$ in water and then stretched to a temporary shape. The nanofiber was reheated at $60^{\circ} \mathrm{C}$, which led to shape recovery. The formation of nanofibers and their morphologies before deformation, during formation of the temporary shape, and after shape recovery were observed by SEM. The surface and cross-section of the shape memory fiber were observed. From the magnified cross-sectional and top-view images, the diameters of the nanofiber before deformation, during formation of the temporary shape, and after shape recovery were calculated. The orientation of the nanofibers before and after deformation, and after shape recovery was analyzed, and the orientation images were created by Image J software using the plugin orientation J to obtain direction distribution maps and their histograms (Figure S6). The shape recovery rate was calculated by cutting the shape memory nanofiber to $1 \mathrm{~cm}$ length and comparing the length of the nanofiber before deformation and shape recovery $(\mathrm{n}=3)$. The nanofiber was heated at $60^{\circ} \mathrm{C}$ in water, and then stretched to a certain elongation degree $(200,300$, or $400 \%)$. The nanofiber was again placed in water at $60^{\circ} \mathrm{C}$ for shape memory recovery. The recovery stress of PCL-9.2 nanofiber was also measured through a tensile test (EZ-S, SHIMADZU, Kyoto, Japan). At first, the sample was extended to 150\% elongation over $60^{\circ} \mathrm{C}$ in chamber (M-600FN, TAITEC, Saitama, Japan) and then, the sample was cooled down to $0{ }^{\circ} \mathrm{C}$. The sample was maintained at the constant deformation for 1 hour. Subsequently, the sample was reheated to $60^{\circ} \mathrm{C}$ and the stress stored in the sample was released. The largest value was taken as the representative of the recovery stress at this strain.

\subsection{Cell culture on nanofiber mesh}


Before starting the hMSC culture, the original and temporarily stretched electrospun nanofibers with a soft:hard segment ratio of $1: 7$ were sterilized by UV irradiation for $15 \mathrm{~min}$. The nanofibers were coated with $20 \mu \mathrm{g} \mathrm{mL}-1$ fibronectin for $1 \mathrm{~h}$ at $37^{\circ} \mathrm{C}$. After washing with PBS, hMSCs were seeded at a density of 5000 cells $\mathrm{cm}^{-2}$ on the sterilized nanofiber and cultured in a MSC growth medium (MSCGMTM, Lonza, Basel, Switzerland)) for 1 day. The hMSCs on the nanofibers were fixed by immersing in $4 \%$ paraformaldehyde for $15 \mathrm{~min}$ and permeabilized with $0.1 \%$ Triton X-100 for $5 \mathrm{~min}$ at room temperature. To visualize F-actin and the nucleus, the cells were treated with rhodamine phalloidin for $30 \mathrm{~min}$ and DAPI for $5 \mathrm{~min}$ in the dark at room temperature. Finally, the cell morphology on the nanofiber meshes was imaged using a fluorescence microscope (ECLIPSE Ti2, Nikon, Tokyo, Japan). To visualize cellular alignment on nanofiber substrates, we prepared a thin nanofiber mesh and seeded cells on it. Then, the fluorescence and phase contrast images were taken for cells and nanofibers, respectively. Finally, two images were merged.

\section{Conclusions}

We reported a novel strategy for the facile production of shape-memory nanofiber meshes. As demonstrated, smooth fibrous structures without beads were formed from PCL-based polyurethanes with different hard-to-soft segment ratios. The original nanofibrous structure easily deformed into a temporary shape, and recovered its original structure when the sample was reheated. A significantly high recovery rate $(>90 \%)$ was achieved even when the mesh was deformed up to $400 \%$. Further, hMSCs aligned well along the fiber orientation when they were cultured on the meshes. Owing to their good biocompatibility, the proposed shape-memory nanofiber system would provide an opportunity to investigate the influence of dynamic changes in matrix structures on cell behaviors. Moreover, another advantage of electrospinning is the possibility of encapsulating drugs in the fibers.

Supplementary Materials: Supplementary materials are available online.

Author Contributions: Conceptualization, M.E.; Methodology, K.U.; Software, K.U, E.N., and K.T.; Formal Analysis, K.T.; Investigation, K.T.; Resources, M.E.; Data Curation, K.T. and E.N.; Writing-Original Draft Preparation, E.N.; Writing-Review \& Editing, K.U., M.E., and A.K.; Supervision, M.E..; Project Administration, M.E.; Funding Acquisition, M.E.

Funding: This research was funded by JSPS KAKENHI (Grant Number 264086 and 26750152).

Acknowledgments: The authors thank the support from Namiki Foundry at National Institute for Materials Science (NIMS) for SEM measurements.

Conflicts of Interest: The authors declare no conflict of interest.

\section{Abbreviations}

$\begin{array}{ll}\text { PCL } & \text { Poly( } \varepsilon \text {-caprolactone) } \\ \text { SMP } & \text { Shape memory polymer } \\ \text { HDI } & \text { Hexamethylene diisocyanate } \\ \text { BD } & \text { 1,4-Butanediol } \\ \text { hMSCs } & \text { Human mesenchymal stem cells } \\ \text { ECM } & \text { Extracellular matrix }\end{array}$

\section{References}


1. Gall, K.; Dunn, M. L.; Liu, Y.; Finch, D.; Lake, M.; Munshi, N. A., Shape memory polymer nanocomposites. Acta Biomaterialia 2002, 50, 5115-5126.

2. Lendlein, A.; Langer, R., Biodegradable, elastic shape-memory polymers for potential biomedical applications. Science 2002, 296, 1673-1676.

3. Nagahama, K.; Ueda, Y.; Ouchi, T.; Ohya, Y., Biodegradable shape-memory polymers exhibiting shpar thermal transitions and controlled drug release. Biomacromolecules 2009, 10, 1789-1794.

4. Hu, J.; Zhu, Y.; Huang, H.; Lu, J., Recent advances in shape-memory polymers: Structure, mechanism, functionality, modeling and applications. Prog Mater Sci 2012, 37 (12), 1720-1763.

5. Liu, Y.; Du, H.; Liu, L.; Leng, J., Shape memory polymers and their composites in aerospace applications: a review. Smart Mater Struct 2014, 23 (2), 023001.

6. Wang, J.; Quach, A.; Brasch, M. E.; Turner, C. E.; Henderson, J. H., On-command on/off switching of progenitor cell and cancer cell polarized motility and aligned morphology via a cytocompatible shape memory polymer scaffold. Biomaterials 2017, 140, 150-161.

7. Tseng, L. F.; Mather, P. T.; Henderson, J. H., Shape-memory-actuated change in scaffold fiber alignment directs stem cell morphology. Acta Biomater 2013, 9 (11), 8790-801.

8. Hsu, S.-H.; Hung, K.-C.; Lin, Y.-Y.; Su, C.-H.; Yeh, H.-Y.; Jeng, U. S.; Lu, C.-Y.; Dai, S. A.; Fu, W.-E.; Lin, J.-C., Water-based synthesis and processing of novel biodegradable elastomers for medical applications. J Mater Chem B 2014, 2 (31), 5083-5092.

9. Chien, Y. C.; Chuang, W. T.; Jeng, U. S.; Hsu, S. H., Preparation, Characterization, and Mechanism for Biodegradable and Biocompatible Polyurethane Shape Memory Elastomers. ACS Appl Mater Interfaces 2017, 9 (6), 5419-5429.

10. Takahashi, T.; Hayashi, N.; Hayashi, S., Structure and Properties of Shape-Memory Polyurethane

Block Copolymers. J Appl Polym Sci 1996, 60, 1061-1069.

11. Leng, J.; Lan, X.; Liu, Y.; Du, S., Shape-memory polymers and their composites: Stimulus methods and applications. Prog Mater Sci 2011, 56 (7), 1077-1135.

12. Ebara, M.; Uto, K.; Idota, N.; Hoffman, J. M.; Aoyagi, T., Shape-memory surface with dynamically tunable nanogeometry activated by body heat. Adv Mater 2012, 24 (2), 273-8.

13. Ebara, M.; Akimoto, M.; Uto, K.; Shiba, K.; Yoshikawa, G.; Aoyagi, T., Focus on the interlude between topographic transition and cell response on shape-memory surfaces. Polymer 2014, 55 (23), 5961-5968.

14. Ebara, M.; Uto, K.; Idota, N.; Hoffman, J. M.; Aoyagi, T., The taming of the cell: shape-memory nanopatterns direct cell orientation. Int J Nanomedicine 2014, 9 Suppl 1, 117-26.

15. Uto, K.; Ebara, M., Magnetic-Responsive Microparticles that Switch Shape at $37{ }^{\circ} \mathrm{C}$. Applied Sciences 2017, 7 (11), 1203.

16. Uto, K.; Mano, S. S.; Aoyagi, T.; Ebara, M., Substrate Fluidity Regulates Cell Adhesion and Morphology on Poly( $\varepsilon-$ caprolactone)-Based Materials. ACS Biomaterials Science \& Engineering 2016, 2 (3), 446-453.

17. Uto, K.; Aoyagi, T.; DeForest, C. A.; Hoffman, A. S.; Ebara, M., A Combinational Effect of "Bulk" and "Surface" Shape-Memory Transitions on the Regulation of Cell Alignment. Advanced Healthcare Materials 2017, 6 (9).

18. Cha, D.; Kim, H. Y.; Lee, K. H.; Jung, Y. C.; Cho, J. W.; Chun, B. C., Electrospun nonwovens of shape-memory polyurethane block copolymers. J Appl Polym Sci 2005, 96 (2), 460-465.

19. Kotek, R., Recent advances in polymer fibers. Polym Rev 2008, 48 (2), 221-229.

20. Zhuo, H.; Hu, J.; Chen, S., Electrospun polyurethane nanofibres having shape memory effect. Mater Lett 2008, 62 (14), 2074-2076.

21. Chung, S. E.; Park, C. H.; Yu, W.-R.; Kang, T. J., Thermoresponsive shape memory characteristics of polyurethane electrospun web. J Appl Polym Sci 2011, 120 (1), 492-500. 
22. Okada, T.; Niiyama, E.; Uto, K.; Aoyagi, T.; Ebara, M., Inactivated sendai virus (HVJ-E) immobilized electrospun nanofiber for cancer therapy. Materials 2015, 9 (1), 12.

23. Garrett, R.; Niiyama, E.; Kotsuchibashi, Y.; Uto, K.; Ebara, M., Biodegradable nanofiber for delivery of immunomodulating agent in the treatment of basal cell carcinoma. Fibers 2015, 3 (4), 478-490.

24. Stitzel, J.; Liu, J.; Lee, S. J.; Komura, M.; Berry, J.; Soker, S.; Lim, G.; Van Dyke, M.; Czerw, R.; Yoo, J. J.; Atala, A., Controlled fabrication of a biological vascular substitute. Biomaterials 2006, 27 (7), 1088-94.

25. Stankus, J. J.; Guan, J.; Fujimoto, K.; Wagner, W. R., Microintegrating smooth muscle cells into a biodegradable, elastomeric fiber matrix. Biomaterials 2006, 27 (5), 735-44.

26. Xu, C.; Inai, R.; Kotaki, M.; Ramakrishna, S., Electrospun nanofiber fabrication as synthetic extracelular matrix and its potential for vascular tissue engineering. Tissue Eng 2004, 10, 11 60-1168.

27. Riboldi, S. A.; Sampaolesi, M.; Neuenschwander, P.; Cossu, G.; Mantero, S., Electrospun degradable polyesterurethane membranes: potential scaffolds for skeletal muscle tissue engineering. Biomaterials 2005, 26 (22), 460615.

28. Shields, K. J.; Beckman, M. J.; Bowlin, G. L.; Wayne, J. S., Mechanical properties and cellular proliferation of electrospun collagen type. Tissue Eng 2004, 10, 1510-1517.

29. Yang, F.; Murugan, R.; Wang, S.; Ramakrishna, S., Electrospinning of nano/micro scale poly(L-lactic acid) aligned fibers and their potential in neural tissue engineering. Biomaterials 2005, 26 (15), 2603-10.

30. Jin, H., Human bone marrow stromal cell responses on electrospun silk fibroin mats. Biomaterials 2004, 25 (6), 1039 1047.

31. Li, W. J.; Tuli, R.; Huang, X.; Laquerriere, P.; Tuan, R. S., Multilineage differentiation of human mesenchymal stem cells in a three-dimensional nanofibrous scaffold. Biomaterials 2005, 26 (25), 5158-66.

32. Dalby, M. J.; McCloy, D.; Robertson, M.; Agheli, H.; Sutherland, D.; Affrossman, S.; Oreffo, R. O., Osteoprogenitor response to semi-ordered and random nanotopographies. Biomaterials 2006, 27 (15), 2980-7.

33. Silva, G. A.; Czeisler, C.; Niece, K. L.; Beniash, E.; Harrington, D. A.; Kessler, J. A.; Stupp, S. 1., Selective Differetiation of Neural progenitor cells by high-epitope density nanofibers. Science 2004, 303, 1352-1355.

34. Curtis, A.; Wilkinson, C., Nantotechniquies and approaches in biotechnology. Trends Biotechnol 2001, 19, 97-101.

35. Mengsteab, P. Y.; Uto, K.; Smith, A. S.; Frankel, S.; Fisher, E.; Nawas, Z.; Macadangdang, J.; Ebara, M.; Kim, D. H., Spatiotemporal control of cardiac anisotropy using dynamic nanotopographic cues. Biomaterials 2016, 86, 1-10.

36. Matsumoto, H.; Ishiguro, T.; Konosu, Y.; Minagawa, M.; Tanioka, A.; Richau, K.; Kratz, K.; Lendlein, A., Shapememory properties of electrospun non-woven fabrics prepared from degradable polyesterurethanes containing poly $(\omega-$ pentadecalactone) hard segments. Eur Polym J 2012, 48 (11), 1866-1874.

37. Fejos, M.; Molnar, K.; Karger-Kocsis, J., Epoxy/Polycaprolactone Systems with Triple-Shape Memory Effect: Electrospun Nanoweb with and without Graphene Versus Co-Continuous Morphology. Materials 2013, 6 (10), $4489-4504$. 38. Ji, F.; Zhu, Y.; Hu, J.; Liu, Y.; Yeung, L.; Ye, G., Smart polymer fibers with shape memory effect. Smart Mater Struct 2006, 15 (6), 1547-1554.

39. Barmouz, M.; Behravesh, A. H., Shape memory behaviors in cylindrical shell PLA/TPU-cellulose nanofiber bio-nanocomposites: Analytical and experimental assessment. Composites: Part A 2017, 101, 160-172.

40. Kawaguchi, K.; Iijima, M.; Miyakawa, H.; Ohta, M.; Muguruma, T.; Endo, K.; Nakazawa, F.; Mizoguchi, I., Effects of chitosan fiber addition on the properties of polyurethane with thermo-responsive shape memory. CHITOSAN FIBER/THERMOPLASTIC POLYURETHANE COMPOSITE MATERIALS 2017, 105, 1151-1156.

41. Maeda, T.; Kim, Y. J.; Aoyagi, T.; Ebara, M., The design of temperature-responsive nanofiber meshes for cell storage applications. Fibers 2017, 5 (1), 13.

42. Ping, P.; Wang, W.; Chen, X.; Jing, X., Poly(e-caprolactone) polyurethane and its shape-memory property. Biomacromolecules 2004, 6, 587-592. 
43. Van Tam, J. K.; Uto, K.; Ebara, M.; Pagliari, S.; Forte, G.; Aoyagi, T., Mesenchymal stem cell adhesion but not plasticity is affected by high substrate stiffness. Sci Technol Adv Mater 2012, 13 (6).

44. Li, G.; Zhang, P., A self-healing particulate composite reinforced with strain hardened short shape memory polymer fibers. Polymer 2013, 54 (18), 5075-5086. 